\title{
Fluctuations and Fermi-Dirac Correlations in $e^{+} e^{-}$-annihilation
}

\author{
Gösta Gustafson \\ Lund University, Dept. of Theoretical Physics, Sölvegatan 14A, S-223 62 Lund, Sweden
}

Received on 6 November, 2006

\begin{abstract}
In this talk I first present a short review of fluctuations in $e^{+} e^{-}$-annihilations. I then describe some new results on FD correlations. Experimental analyses of $p p$ and $\Lambda \Lambda$ correlations indicate a very small production radius. This result relies very strongly on comparisons with $\mathrm{MC}$ simulations. A study of the approximations and uncertainties in these simulations implies that it is premature to draw such a conclusion from the data.
\end{abstract}

Keywords: Fluctuations; Correlations

\section{FLUCTUATIONS}

In bremsstrahlung in QED, the emission of a photon does not change the current for subsequent emissions. This implies that the photon multiplicity is described by a Poisson distribution. As the gluons carry colour charge, the emission of a gluon in QCD changes the current relevant for the subsequent emissions. An initial hard gluon will radiate many softer gluons. This leads to a cascade with an exponential growth in multiplicity and to large fluctuations. The multiplicity distribution is approximately satisfying KNO scaling, with a width which is proportional to the average multiplicity. This short review is divided in three parts:
A. Multiplicity distribution for partons
B. Effects of hadronization
C. Fractal structures

\section{A. Multiplicity distribution for partons}

In $e^{+} e^{-}$-ann. the emission of a gluon from a $q \bar{q}$ pair is a coherent emission from e.g. a red and an anti-red colour charge. The result corresponds to a colour dipole with the following distribution (in the leading log approximation):

$$
d N=\bar{\alpha}\left(k_{\perp}^{2}\right) \frac{d k_{\perp}^{2}}{k_{\perp}^{2}} d y, \text { with } \bar{\alpha}=\frac{3 \alpha_{s}}{2 \pi} .
$$

The phase space is a triangular region in $\left(y, \ln k_{\perp}^{2}\right)$-space given by $|y|<\ln \left(s / k_{\perp}^{2}\right) / 2$, and within this triangle the density is just given by $\bar{\alpha}$. The emission of a second (softer) gluon is given by two dipoles, one between the quark and the first gluon, and one between this gluon and the antiquark. The phase space is enlarged compared to that for the first gluon, and corresponds to the folded surface shown in Fig. 1a. For subsequent gluons the phase space is further increased, and corresponds to the fractal surface in Fig. 1b.

We let $P(N, L=\ln s)$ denote the distribution in the number, $N$, of dipoles (which neglecting $q \bar{q}$ pair creation is equal to the number of gluons plus one). The Laplace transform $P(\gamma, L)$ is given by

$$
\mathcal{P}(\gamma, L) \equiv \sum_{N} e^{-\gamma N} P(N, L)
$$

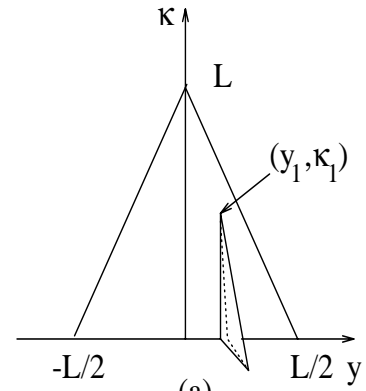

(a)

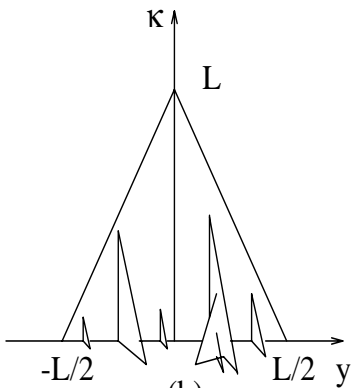

(b)
FIG. 1: The phase space for gluon emission in $e^{+} e^{-}$-ann. is a triangular region in the $\left(y, \kappa=\ln k_{\perp}^{2}\right)$-plane. The height of the triangle is given by $L=\ln s$. When one gluon is emitted at $\left(y_{1}, \kappa_{1}\right)$ a second gluon with smaller $k_{\perp}$ can be emitted in the larger phase space shown in Fig. (a). Further softer emissions give the fractal phase space in Fig. (b).

It is then relatively easy to derive the relation (see e.g. ref. [1])

$$
\frac{d^{2} \ln \mathcal{P}}{d L^{2}}=\frac{\alpha_{0}}{L}(\mathcal{P}-1), \text { where } \bar{\alpha} \equiv \frac{\alpha_{0}}{L} .
$$

Expanding eq. (2) in a Taylor series in $\gamma$ gives

$$
\mathcal{P}=1-\gamma\langle N\rangle+\frac{1}{2} \gamma^{2}\left\langle N^{2}\right\rangle+\ldots
$$

Inserted in eq. (3) this also implies the following equations for the average and the variance of the multiplicity distribution:

$$
\begin{gathered}
\frac{d^{2}}{d L^{2}}\langle N\rangle=\frac{\alpha_{0}}{L}\langle N\rangle \\
\frac{d^{2}}{d L^{2}}\left(\left\langle N^{2}\right\rangle-\langle N\rangle^{2}\right)=\frac{\alpha_{0}}{L}\left\langle N^{2}\right\rangle .
\end{gathered}
$$

The solutions to these equations are Bessel functions, and for high energies we get

$$
\begin{aligned}
\langle N\rangle & \sim L^{1 / 4} \exp \left(2 \sqrt{\alpha_{0} L}\right) \\
V & \equiv\left\langle N^{2}\right\rangle-\langle N\rangle^{2} \approx \frac{1}{3}\langle N\rangle^{2} .
\end{aligned}
$$

We see that the width of the distribution is proportional to $\langle N\rangle$, in agreement with KNO scaling, and thus for large $N$ the distribution is much wider than a Poisson distribution. 
The anomalous dimension is given by a logarithmic derivative. Note that in the literature one can find different definitions, where the derivative is taken with respect either to $\ln s$ or to $\ln W$ :

$$
\begin{gathered}
\gamma_{0}^{(s)} \equiv \frac{d \ln \langle N\rangle}{d \ln s}=\sqrt{\bar{\alpha}}=\sqrt{\frac{3 \alpha_{s}}{2 \pi}} \\
\gamma_{0}^{(W)} \equiv \frac{d \ln \langle N\rangle}{d \ln W}=2 \sqrt{\bar{\alpha}}=\sqrt{\frac{6 \alpha_{s}}{\pi}} .
\end{gathered}
$$

I want here to add a few comments:

- $\langle N\rangle$ depends on the resolution, i.e. on the cut-off for soft emissions. It is here essential to have a cut-off which is defined locally, and not fixed in e.g. the overall cms. In the dipole cascade model this is chosen as $k_{\perp}$ measured in the rest frame of the emitting dipole.

- The running of $\alpha_{s}$ is important. With a constant $\alpha_{s}$ the multiplicity would grow proportional to $\exp (\sqrt{\bar{\alpha}} \cdot L)$. i.e. much faster than the result in eq. (5).

- Non-leading corrections are very large. Terms suppressed by factors $1 / \sqrt{L}$ in the evolution equation give extra powers of $L$ as factors in the solution. Also including NLL terms, the result is sensitive to effects of still higher order. For a more detailed discussion see e.g. ref. [2]

\section{B. Hadronization effects}

In string fragmentation the average hadron multiplicity, $\langle n\rangle$, in a single $q \bar{q}$ system is proportional to $\ln \left(s / s_{0}\right)$, where $s_{0}$ is a scale of order $1 \mathrm{GeV}$. The variance of the fluctuations, $V=\left\langle n^{2}\right\rangle-\langle n\rangle^{2}$, is proportional to $\langle n\rangle$, and the distribution is thus relatively more narrow for higher energies and larger $\langle n\rangle$. For a system of a $q \bar{q}$ pair and a number of gluons we get a set of string pieces with (squared) masses $s_{i, i+1}=\left(q_{i}+q_{i+1}\right)^{2}$, where $q_{i}$ is the momentum of parton $i$ and the partons are ordered in colour. For the average multiplicity we then get

$$
\langle n\rangle \propto \sum \ln \left(s_{i, i+1} / s_{0}\right)
$$

which just corresponds to the length of the baseline of the fractal surface in Fig. 1b.

In eq. (10) the mass of a string piece should not be smaller than $\sqrt{s_{0}}$, as it otherwise would give a negative contribution. It is, however, also possible to define an infrared stable measure, called the $\lambda$-measure, which is insensitive to the cut-off for the perturbative cascade [3]. Simulations show that a high energy even with little gluon radiation and an event at lower energy but more radiation give equally many hadrons if they have the same $\lambda$-value. Thus the multiplicity depends only on the measure $\lambda$, which corresponds to an "effective string length". The probability density $P(\lambda, L)$ satisfies the same evolution equation (3) as $P(N, L)$, only with different boundary conditions. This implies that the distribution in $\lambda$ has the same high energy behaviour as the distribution in dipole multiplicity, eq. (5).
The fluctuations in the hadron multiplicity get contributions from both the perturbative cascade and the soft hadronization, and we get approximately

$$
\frac{V(n, s)}{\langle n(s)\rangle^{2}}=\frac{V(\lambda, s)}{\langle\lambda(s)\rangle^{2}}+\frac{V(n,\langle\lambda\rangle)}{\langle n(\langle\lambda\rangle)\rangle^{2}} .
$$

Here the first term corresponds to the fluctuations in the cascade and the second one to those in the hadronization process. At high energy the first contribution dominates, while the second is larger at energies below $40-50 \mathrm{GeV}$. In ref. [3] it is shown that the total result satisfies KNO scaling with $V=$ const $\cdot\langle n\rangle^{2}$, although this is not the case for any of the contributions separately below the top LEP energy. At higher energies the hadronization contribution can be neglected.

\section{Fractal structures and intermittency}

The baseline of the surface in Fig. 1b looks like a Koch snowflake curve. Looking at the curve with a coarser resolution means that only emissions with $k_{\perp}>k_{\perp}$ res are included. This corresponds to cutting the surface in Fig. 1b at a higher level, corresponding to $\ln k_{\perp \text { res }}^{2}$. The length of this curve satisfies again the same evolution equation. The boundary conditions are different, and with the notations $L=\ln s$ and $\kappa=\ln k_{\perp}^{2}$ the length will be proportional to the expression

$$
\text { Length } \sim L^{1 / 4} \kappa_{\mathrm{res}}^{3 / 4} \exp \left(2 \sqrt{\alpha_{0} L}-2 \sqrt{\alpha_{0} \kappa_{\mathrm{res}}}\right)
$$

It is also possible to define a (multi)fractal dimension for this curve

$$
D=1-\frac{d \ln \text { length }}{d \ln \left(\kappa_{\mathrm{res}}\right)}=1+\sqrt{\frac{\alpha_{0}}{\kappa_{\mathrm{res}}}}=1+\gamma_{0}^{(s)}
$$

(It is called a multifractal as the dimension varies with the resolution $\kappa_{\text {res. }}$ )

Fluctuations in small phase space regions, e.g. slices in rapidity $\Delta y$, can be described by the scaled moments

$$
\begin{aligned}
C_{q} & \equiv \frac{\left\langle n^{q}\right\rangle}{\langle n\rangle^{q}} \\
F_{q} & \equiv \frac{\langle n(n-1) \ldots(n-q+1)\rangle}{\langle n\rangle^{q}}
\end{aligned}
$$

If the total phase space is divided in $M$ equal pieces (which gives $\Delta y=Y / M)$ a scaling behaviour $F_{q} \propto M^{\phi_{q}}$ is interpreted as an signal for intermittency. This can be associated with a Renyi (multifractal) dimension

$$
D_{q} \equiv 1-d_{q} \equiv 1-\frac{\phi_{q}}{q-1}
$$

In the perturbative cascade these fluctuations in small bins are dominated by the possibility to have the tip of a jet within one bin. At asymptotic energies and large $q$ we then get the result [5-8]

$$
D_{q} \approx \frac{q}{q-1} \sqrt{\frac{6 \alpha_{s}}{\pi}}
$$



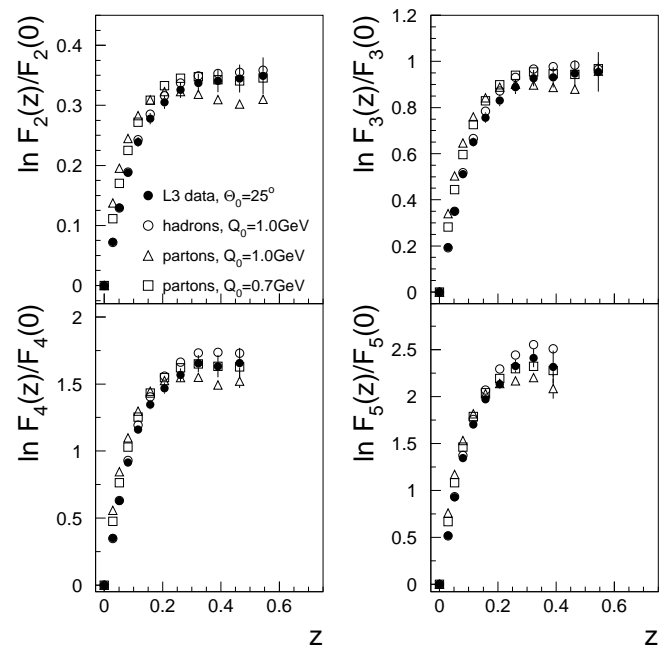

FIG. 2: L3 results for ratios of factorial moments in cones around a jet axis, $F_{q}(z) / F_{q}(0)$, together with JETSET 7.4 PS predictions on partonic and hadronic levels. For details see ref. [9].
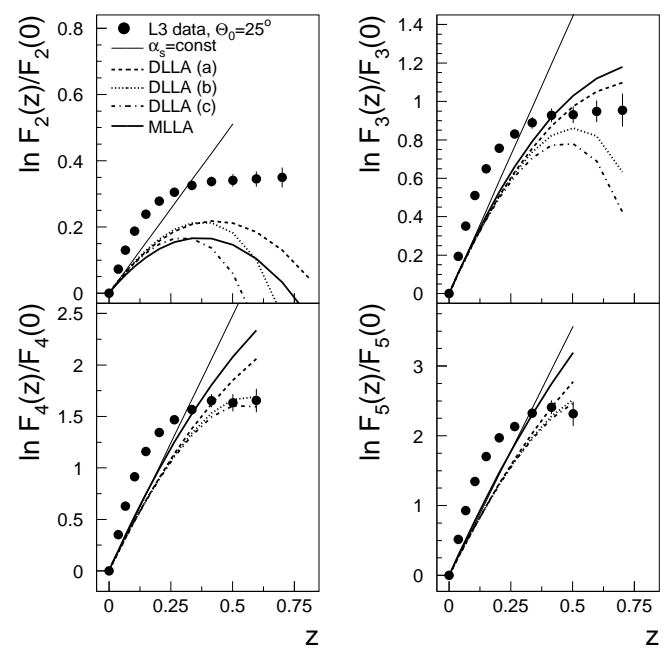

FIG. 3: Analytic QCD predictions for $\Lambda=0.16 \mathrm{GeV}$. The curves show results for $\alpha_{s}=$ const., 3 different DLL approximations and a MLL result. For details see ref. [9].

As for the distributions in the full phase space, there are large corrections from non-leading effects and from hadronization. Therefore the analytic results have had limited success in comparisons with experimental data. As examples Figs. 2 and 3 show results from the L3 collaboration at LEP [9] for factorial moments in regions separated by cones with opening angels $\Theta_{0}-\Theta$ and $\Theta_{0}+\Theta$ around a jet axis. The variable $z$ is proportional to $\ln \left(\Theta_{0} / \Theta\right)$. We see that although MC programs work quite well (Fig. 2), the analytic calculations do not reproduce the data (Fig. 3). We note that different NLL calculations give quite different results. These calculations have different approximations for NNLL contributions, which shows that the higher order corrections cannot be neglected. (It was early realised that BE correlations give an important contribution to the multiplicity moments, and in the L3 analysis this effect is removed from the data.)

It is also possible to define the $\lambda$-measure for a hadronic state instead of a parton state, and it appears that analytic results for the $\lambda$-measure are closer to the corresponding MCresults [6]. No comparisons with experimental data have been presented until now for such an analysis.

\section{Summary}

The gluon self coupling implies a fast growth of multiplicity and large fluctuations. In LLA we get the following asymptotic results:

$$
\begin{aligned}
\langle N\rangle & \sim \exp \left(2 \sqrt{\alpha_{0} \ln s} ; \quad \alpha_{0} \equiv \frac{3 \alpha_{s}\left(Q^{2}\right)}{2 \pi} \ln Q^{2}\right. \\
V & =\frac{1}{3}\langle N\rangle^{2} ; \quad \gamma_{0} \equiv \frac{d \ln \langle N\rangle}{d \ln s}=\sqrt{\frac{3 \alpha_{s}}{2 \pi}}
\end{aligned}
$$

The QCD cascade has a fractal structure. The multiplicity in small phase space intervals shows intermittent features with a multifractal (Renyi) dimension $D_{q} \sim 2 \gamma_{0}^{(s)}$, and the curve with the $\lambda$-measure has the dimension $1+\gamma_{0}^{(s)}$.

However, energy-momentum conservation and other nonleading effects are very important. NLL calculations give very different results if they differ at NNLL order. Hard emissions are the most important, but matrix elements factorize only for soft emissions. Therefore analytic calculations have in many cases met limited success. MC programs work, however, generally quite well.

\section{FERMI-DIRAC CORRELATIONS IN $p p$ AND $\Lambda \Lambda$ PAIRS}

\section{A. Momentum correlations}

The results on baryon correlations presented here are obtained in collaboration with R.M. Durán Delgado and L. Lönnblad [10].

Fermion correlation functions are usually fitted to the form

$$
C(Q)=N\left\{1-\lambda e^{-R^{2} Q^{2}}\right\} .
$$

The quantity $R$ is here interpreted as the radius of the production region for the particle pair. The different LEP experiments have measured momentum correlations between $\Lambda \Lambda$ and/or $\bar{p} \bar{p}$ pairs, and obtained production radii around $0.15 \mathrm{fm}$, which is much smaller than the proton radius [11-14].

In experiments the correlation function $C(Q)$ is determined by comparing the observed number of pairs, $N(Q)$, with a reference sample, $N_{\text {ref }}(Q)$ :

$$
C(Q)=\frac{N(Q)}{N_{\mathrm{ref}}(Q)} .
$$

The important question is how to construct the reference sample. Different methods have been applied: 
- MC generation. This is model dependent.

- Mixed events. The event structure is changed by gluon emission, which makes it difficult to construct a sample of events with similar structure.

A common method to reduce the problems is to take a double ratio:

$$
C(Q)=\frac{N(Q)}{N_{\operatorname{mix}}(Q)} / \frac{M C(Q)}{M C_{\operatorname{mix}}(Q)}
$$

Here the sample of Monte Carlo events, $M C(Q)$, should be generated without including FD correlations in the model. It is argued that the effect of different event types should be similar in the real data and the MC events, and therefore be reduced in the ratio.

The mixed pairs depend only on inclusive spectra, and the MC programs are tuned to reproduce the inclusive spectra with good precision. This implies that the tuning also gives

$$
N_{\text {mix }}(Q) \approx M C_{\text {mix }}(Q)
$$

which implies that

$$
C(Q) \approx \frac{N(Q)}{M C(Q)}
$$

Consequently the result depends very critically on a realistic $M C$, also if the double ratio is used in the analysis.

In the Lund string hadronization model the colour string normally breaks by $q \bar{q}$ pair production. The ordering of the hadrons along the string, the rank ordering, agrees on average with the ordering in rapidity, with an average separation, $\Delta y$, of the order of half a unit in rapidity. A baryon-antibaryon pair can be produced when a breakup is generated by a diquarkantidiquark pair, with quark-antiquark pairs on either side. In this picture two baryons must always be separated by at least one antibaryon (and normally also with one or more mesons). This will give a strong anti-correlation between two baryons in rapidity and in momentum. This is seen in Fig. 4, which shows correlations in $p p$ and $p \bar{p}$ pairs. We see that there is a positive correlation between protons and antiprotons, which are neighbours in rank, but a negative correlation between two protons.

In the model there is also a correlation between momentum and space coordinates for the produced hadrons. Thus two identical baryons are (in the model) well separated also in coordinate space, and we would from this picture expect Fermi-Dirac correlations to correspond to a radius $\sim 2-3 \mathrm{fm}$.

We see that the range for the $p p$ correlation in Fig. 4 is given by $Q \sim 1.5 \mathrm{GeV} \sim 1 /(0.15 \mathrm{fm})$. We note that this corresponds exactly to the correlation length reported in the experiments, although in this model the production radius is very much larger. The strength of the correlation is, however, smaller in the MC than in the data.

This raises the question: Is the difference between data and $M C$ really a FD effect, or could the MC underestimate the strength of the correlation?

There are a number of sources for uncertainty in the MC:

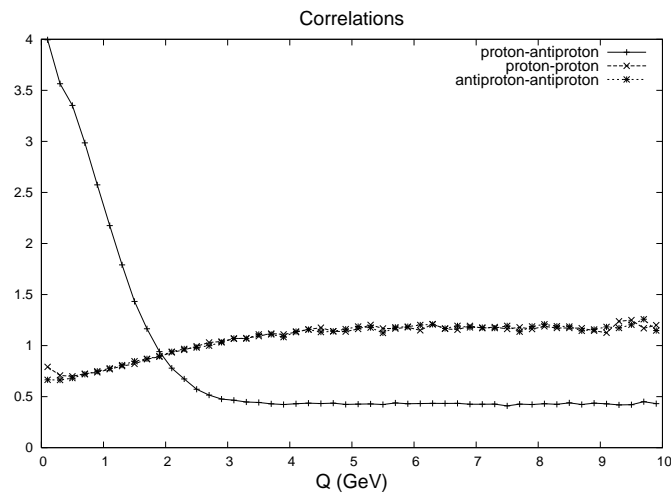

FIG. 4: $\mathrm{MC}$ results for the ratio $C_{M C}(Q)=M C(Q) / M C_{\text {mix }}(Q)$ for $p p-(\times)$ and $p \bar{p}$-pairs $(+)$.

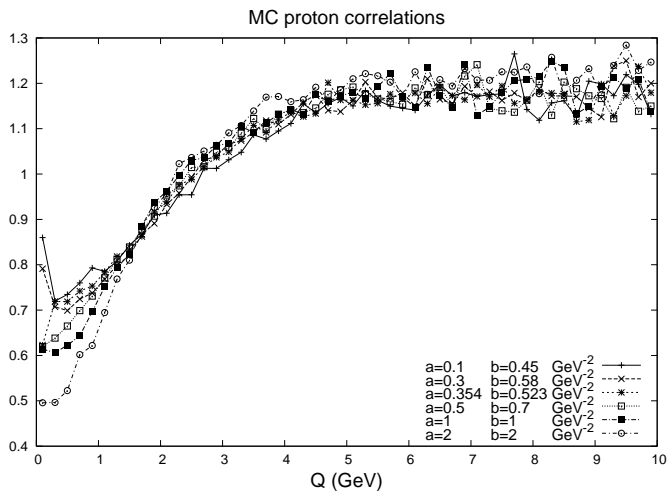

FIG. 5: The ratio $C_{M C}(Q)=M C(Q) / M C_{\text {mix }}(Q)$ for different values of the parameters $a$ and $b$. Larger $(a, b)$-values give a stronger correlation and a deeper dip for small $Q$.

1) There are two fundamental parameters, $a$ and $b$, in the Lund model "splitting function"

$$
f(z) \propto(-z)^{a} e^{-b m^{2} / z}
$$

The hadron multiplicity depends essentially on the ratio $(a+$ $1) / b$, This ratio is therefore well determined by experiments, but $a$ and $b$ separately are more uncertain. Small values of $a$ and $b$ correspond to a wide distribution $f(z)$, and a wide distribution in the separation, $\Delta y$, between hadrons which are neighbours in rank. Large values of $a$ and $b$ imply a narrow $\Delta y$ - distribution and therefore lower probability for two particles to be close in momentum space. The effect of varying $a$ and $b$ keeping the multiplicity unchanged is shown in Fig. 5.

2) The parameter $b$ is a universal constant, but $a$ may be different for baryons, although data are well fitted by a universal $a$-value. This gives some extra uncertainty.

3) The MC does not exactly reproduce the Lund hadronization model. The splitting function in eq. (24) gives a correct result when the remaining energy in the system is large. To minimise the error at the end of the cascade, when the energy is small, the MC cuts off hadrons from both ends randomly, and joins the two ends when the remaining mass is small. This 


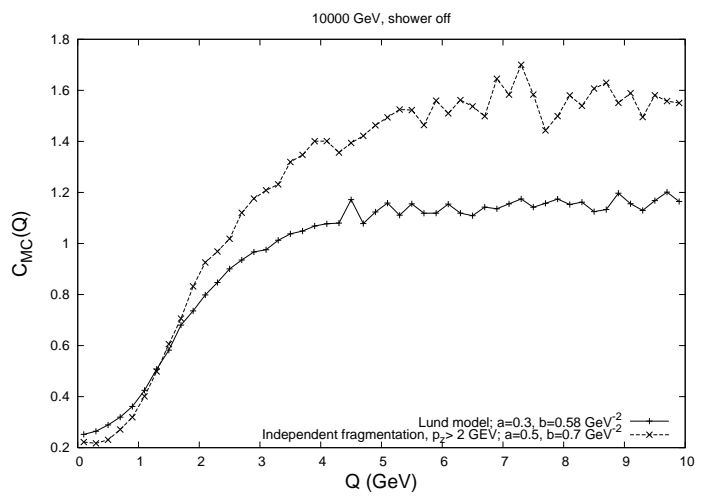

FIG. 6: A single jet without a junction has a large dip in $C_{M C}(Q)=$ $M C(Q) / M C_{\operatorname{mix}}(Q)$ for small $Q(\times)$. This correlation is reduced by the approximate treatment in the $\mathrm{MC}$ of the small mass systems close to the "junction" (+).

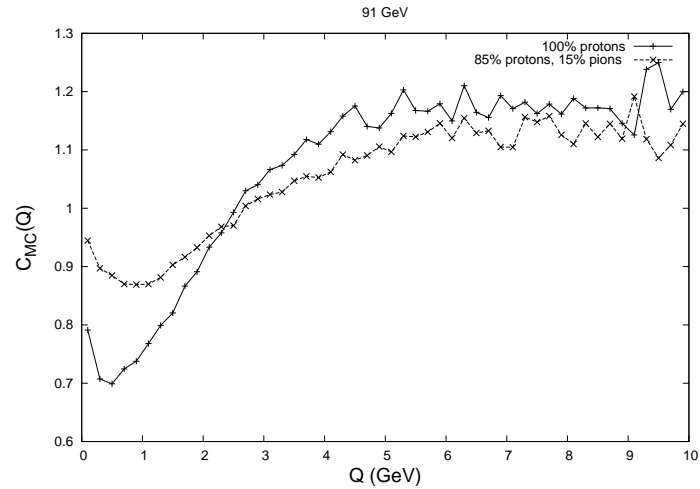

FIG. 7: $C_{M C}(Q)=M C(Q) / M C_{\text {mix }}(Q)$ with $(+)$ and without $(\times)$ a $15 \%$ admixture of pions.

works well for inclusive distributions, but implies that the correlations do not correspond to the model prediction for particles close to the "junction". Fig. 6 shows results for a single jet without a junction compared with the standard result.

4) Gluon emissions imply that straight string pieces are small compared to the mass of a $B \bar{B} B$ system. This gives also extra uncertainty.

5) Pion correlations are not perfectly reproduced by the MC. As an example the DELPHI antiproton sample contains $15 \%$ pions. Fig. 7 shows results with and without a $15 \%$ pion admixture. We see that an error in the simulation of the pion-pion or pion-proton correlations also affects the estimated proton-proton correlations.

In summary we see that there are many effects which make the $\mathrm{MC}$ predictions for $p p$ or $\Lambda \Lambda$ correlations quite uncertain. As the experimental determination of the correlations rely so strongly on a correct $\mathrm{MC}$, it is therfore at present premature to conclude that the production radius has the very small value around $0.15 \mathrm{fm}$.

\section{B. Spin-spin correlations}

$\Lambda$ particles reveal their spin in the orientation of their decay products. This has been used to study $\Lambda \Lambda$ correlations without the need for a comparison with $\mathrm{MC}$ results. A $\Lambda \Lambda$ pair with total spin 1 must have an antisymmetric spacial wave function and is therefore expected to show a suppression for small relative momenta $Q . \Lambda \Lambda$ pairs with total spin 0 has a symmetric spacial wave function, and should therefore show an enhancement for small $Q$, similar to the correlation for bosons. Therefore one expects pairs with small $Q$-values to be dominantly $S=0$. Analyses at LEP $[11,15,16]$ do indicate such an effect. They are however based on rather low statistics, and the errors are presently too large for any definite conclusion about the strength and range of the effect.

\section{Summary}

A production radius $R \sim 0.15 \mathrm{fm}$ for baryon pairs is not consistent with the conventional picture of string fragmentation.

Experimental results for $p p$ and $\Lambda \Lambda$ correlations depend sensitively on a reliable MC.

The observed (anti-)correlation has the same range in $Q$ as the correlation in the $\mathrm{MC}$, but is stronger.

Uncertainties in the MC implementation are large.

Conclusion: It is therefore premature to claim evidence for a new production mechanism.
[1] B. Andersson, P. Dahlqvist, and G. Gustafson, Phys. Lett. B 214, 604 (1988)

[2] G. Gustafson and M. Olsson, Nucl. Phys. B 406, 293 (1993).

[3] B. Andersson, P. Dahlqvist, and G. Gustafson, Z. Phys. C 44, 455 (1989).

[4] B. Andersson, G. Gustafson, and B. Söderberg, Nucl. Phys. B 264, 29 (1986).

[5] P. Dahlqvist, B. Andersson, and G. Gustafson, Nucl. Phys. B 328, 76 (1989)

[6] G. Gustafson and A. Nilsson, Z. Phys. C 52, 533 (1991).

[7] W. Ochs and J. Wosiek, Phys. Lett. B 289, 159 (1992).

[8] W. Ochs and J. Wosiek, Phys. Lett. B 305, 144 (1993).

[9] W. Kittel, S.V. Chekanov, D.J. Mangeol, and W.J. Metzger,
Nucl. Phys. Proc. Suppl. 71, 90 (1999); hep-ex/9712003.

[10] R.M. Durán Delgado, Diploma thesis,Lund preprint LU TP 06-17 (2006), and R.M. Durán Delgado, G. Gustafson, and L. Lönnblad, in preparation.

[11] R. Barate et al. (ALEPH Coll.), Phys. Lett. B 475, 395 (2000).

[12] OPAL Coll., CERN-EP/2001, OPAL-PN486 (2001).

[13] R. Barate et al., (ALEPH Coll.), Phys. Lett. B 611, 66 (2005).

[14] DELPHI Coll., DELPHI 2004-038 CONF-713 (23 June, 2004), DELPHI 2005-010 CONF-730 (30 May, 2005).

[15] G. Alexander et al. (OPAL Coll.), Phys. Lett. B 384, 377 (1996).

[16] T. Lesiak and H. Palka (DELPHI Coll.), CERN-EP/98-114, DELPHI-CONF-176 (1998) 